\title{
Comparative study of controllers for switch power converters using Pspice
}

\author{
Dr.A.R.Garg1, Mrs.SwapnaManurkar ${ }^{2}$ \\ Associate Prof, Electrical Engineering, MBM ENGINEERING College Jodhpur, India ${ }^{1}$ \\ Assistant Prof, Electrical Engineering, SKNSITS, Lonavala, India ${ }^{2}$
}

\begin{abstract}
The present paper talks about the comparison of type1, type 2 and type 3 controllers for isolated switch mode power supplies. The converters selected for study are Buck,Boost,Buck and boost type both in VM and CM. Small signal model is prepared for all the types mentioned above and Bode's plot is drawn followed by stability analysis based on GM and PM. The software used for same purpose is Orcad capture Pspice.
\end{abstract}

Keywords: VM, CM, Small signal model, Stability analysis, GM, PM

\section{INTRODUCTION}

Almost all the electronic circuits, such as signal processing circuits radio receivers, and computers which work on Dc, generally carry small time-varying (AC) signals on top of a constant (DC) bias. These ac signals how so ever small it may be must interfere with dc supply and disturb it's working. This suggests that there must be some method to find out the effect of these small signal perturbations about the bias point.

The process for linearising a non linear system about a bias point is to take partial derivative of the formula expressing the non linear system with respect to all the variables governing the system. The resultant partial derivatives are thereafter can be expressed by physical quantities like inductance, resistance and capacitance. Finally a circuit diagram can be proposed representing the system. Such a small signal models exist for devices like FET's, diodes, electron tubes MOSFET and BJTs and also for switched power converters.

This small signal model gives us the output response of the system when subjected to vary small ac signal over input Dc signal. The resultant response is in the form of Bode's plot that is frequency response of the system and from these plots we can find GM, PM and hence the stability of system for given ac signal. Orcad capture comes with special features to find these values directly by using functions provided in the software.

\section{SMALL SIGNAL MODELING}

Modeling is the representations of physical phenomenon by mathematical means. Thus, modeling process involve use of approximations to neglect small but complicating phenomena, in order to understand what is most important. It is fact that physical models are complex, and their detail analysis can lead to an intractable and useless mathematical mess. Approximate models are thus important tool for designing and gaining physical insight of such models.

The switching ripple is small in well designed converter operating in CCM. Hence, we can ignore this switching ripple and model only underlying ac variations. The purpose of using ac modeling is to predict the presence of low frequency component in the main spectrum of switching frequency .
The analysis of small signal model begins with the determination of the voltage and current waveforms for given topology then writing the equations for specific condition of switch, i.e. either ON or OFF.

Small signal model for Buck and Boost converter is derived in next section.

A Small signal model of buck and boost converter

Consider the switch in position on and off in fig $1 \& 2$ respectively of buck and boost converter
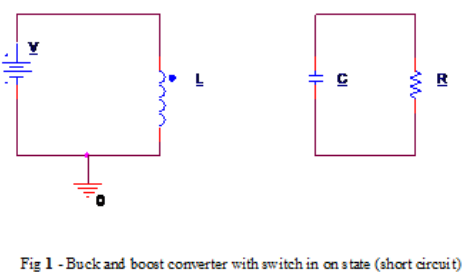

Sw1

$v_{L}(t)=L \frac{d i}{d t}=v_{g}(t)$

$i_{c}(t)=C \frac{d v}{d t}=-\frac{v(t)}{R}$

Make the small signal ripple approximation. But rather than replacing $v_{g}(t)$ and $v(t)$ by their dc values, we now replace them with their low frequency averaged values $<v_{g}(t)>_{T_{S}}$ and $<v(t)>_{T_{S}}$.

So the equations now become

$v_{L}(t)=L \frac{d i}{d t}=<v_{g}(t)>_{T_{S}}$

$i_{c}(t)=C \frac{d v}{d t}=-\frac{<v(t)>T_{S}}{R}$

Sw2

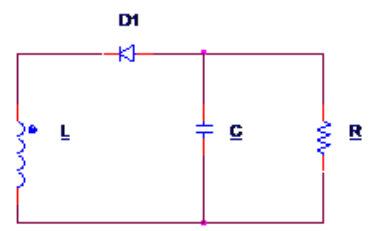

Fig 2 - Buck and boost converter withswitch in off state (open circvit) 
$v_{L}(t)=L \frac{d i}{d t}=v(t)$

$i_{c}(t)=C \frac{d v}{d t}=-i(t)-\frac{v(t)}{R}$

Again replace $=-i(t)$ and $v(t)$ with their averaged values, yields

$$
\begin{aligned}
& v_{L}(t)=L \frac{d i}{d t}=\left\langle v(t)>_{T_{S}}\right. \\
& i_{c}(t)=C \frac{d v}{d t}=-<i(t)>_{T_{S}}-\frac{<v(t)>T_{S}}{R}
\end{aligned}
$$

Combining the two sub intervals as done previous

$$
<v_{L}(t)>_{T_{S}}=\frac{1}{T_{S}} \int_{t}^{t+T_{S}} v_{L}(\tau) d \tau=
$$

$d(t)<v_{g}(t)>_{T_{S}}+d^{\prime}(t)<v(t)>_{T_{S}}$

The equation can be written as

$$
L \frac{d i}{d t}=d(t)<v_{g}(t)>_{T_{S}}+d^{\prime}(t)<v(t)>_{T_{S}}
$$

Averaging the capacitor current waveform

$<i_{c}(t)>=$

$$
\begin{aligned}
& d(t)\left(\frac{<-v(t)>_{T_{S}}}{R}\right)+d^{\prime}(t)\left(-<i(t)>_{T_{S}} \frac{-v(t)}{R}\right)= \\
& \frac{<-v(t)>_{T_{S}}}{R}-<i(t)>_{T_{S}} d^{\prime}(t)--(7) \\
& <i_{g}(t)>_{T_{S}}=d(t)<i(t)>_{T_{S}}+0 d^{\prime}(t)=d(t)< \\
& i(t)>_{T_{S}} \quad--(8)
\end{aligned}
$$

Thus three equations $(6,7 \& 8)$ describing the model are:

$<v_{L}(t)>_{T_{S}}=d(t)<v_{g}(t)>_{T_{S}}+d^{\prime}(t)<v(t)>_{T_{S}}$

$<i_{c}(t)>=\frac{<-v(t)>_{T_{S}}}{R}-<i(t)>_{T_{S}} d^{\prime}(t)$

$<i_{g}(t)>_{T_{S}}=d(t)<i(t)>_{T_{S}}$

All these equations are non linear, so they have to be linearized by constructing a small signal model.

For buck and boost converter it is known from the equation -(3.22) of duty cycle that

$$
\begin{aligned}
& V=-\frac{D}{D^{\prime}} V_{g} \\
& I=-\frac{V}{D^{\prime} R} \\
& I_{g}=D I
\end{aligned}
$$

These equations are derived by assuming that converter is driven at some steady state duty ratio $d(t)=D$, and $V g(t)=V g$. Also from chapter 3 we can say that when all the transients have subsided inductor current, capacitor voltage and the input current will reach the quiescent value.

To construct a small signal ac model at a quiescent operating point $(V, I)$ one assumes that the input voltage $V_{g}(t)$ and duty cycled $(t)$ are equal to some given quiescent value plus small superimposed ac variations $\widehat{V_{g}}(t), \hat{d}(t)$ and hence we can write $<v_{g}(t)>_{T_{S}}=V_{g}(t) \widehat{+V}_{g}(t)$

$d(t)=D+\hat{d}(t)$
In response to these inputs, after any transients have subsided

The averaged inductor current, the average capacitor voltage, and the averaged input current, waveform will be equal to corresponding quiescent values plus some small superimposed ac variationsî $(t), \hat{v}(t), \hat{l}_{g}(t)$

$<\hat{\imath}(t)>_{T_{S}}=I+\hat{\imath}(t)$

$<\hat{v}(t)>_{T_{S}}=V+\hat{v}(t)-(11)$

$<\hat{\imath}_{g}(t)>_{T_{S}}=I_{g}+\hat{\imath}_{g}(t)$

Since ac variations are small in magnitude as compared to the dc quiescent values i.e.

$\left|\widehat{v_{g}}(t)\right|<\left|V_{g}\right|$
$|\hat{d}(t)|<|D|$
$|\hat{\imath}(t)|<|I|$
$|\hat{v}(t)|<|V|$
$\left|\widehat{\iota_{g}}(t)\right|<\left|I_{g}\right|$

Thus the nonlinear equation $6-8$ can be linearized. This is done by inserting equation $9-11$ into $6-8$.

For the inductor equation, following result is obtained $L \frac{d(I+\hat{\imath}(t))}{d t}=(D+\hat{d}(t))\left(V_{g}+\widehat{v_{g}}(t)\right)+\left(D^{\prime}-\right.$ $d t(V+v(t)) \quad--(13)$

After expanding the equation becomes:

$L\left(\frac{d I}{d t}+\frac{d \hat{\imath}}{d t}\right)=\left(D V_{g}+D^{\prime} V\right)+\left(D \hat{v}_{g}(t)+D^{\prime} \hat{v}(t)+\right.$

$V g-V d t+d t v g t-v t--(14)$

In the above equation the first term on the R.H.S. represents Dc term (term contain dc quantities only), second represents $1^{\text {st }}$ order ac term (these terms are linear functions of the ac variations) and the third term is $2^{\text {nd }}$ order ac term (these term contain product of ac quantities, and are therefore nonlinear) respectively.

The derivative of dc quantities is zero, therefore first term on R.H.S. is zero, as derivative of I is zero.

Also it is desired to neglect the nonlinear a terms. By assumption made in equations 12each of the second order nonlinear term is much smaller in magnitude than one or more of the linear first order term. So it can be easily neglected.

Now comparing the terms on L/H.S. and terms on the R.H.S. we are left with.

$L\left(\frac{d \hat{\imath}}{d t}\right)=D \hat{v}_{g}(t)+D^{\prime} \hat{v}(t)+\left(V_{g}-V\right) \hat{d}(t)$

This is small signal linearized equation that describes variation in inductor current.

In the same manner capacitor equation is also linearized. Insert equation $9-11$ into 7 yields,

$c \frac{d(V+\hat{v}(t))}{d t}=\left(D^{\prime}-\hat{d}(t)\right)(I+\hat{\imath}(t))-\frac{(V+\hat{v}(t))}{R}$

Multiplying and collecting terms we obtain,

$c\left(\frac{d V}{d t}+\frac{d \hat{v}(t)}{d t}\right)=\left(-D^{\prime} I-\frac{V}{R}\right)+\left(-D^{\prime} \hat{\imath}(t)-\frac{\hat{v}(t)}{R}+\right.$

$I d t+d(t) i(t)$ 
As done earlier Dc Terms on both the sides are equal and zero, second order terms are neglected and hence we are left with only first order linearized ac terms given by , $c \frac{d \hat{v}(t)}{d t}=-D^{\prime} \hat{\imath}(t)-\frac{\hat{v}(t)}{R}+I \hat{d}(t)$

Now linearized the equation for average input current, $I_{g}+\hat{\imath}_{g}(t)=(D+\hat{d}(t))(I+\hat{\imath}(t))$

Again neglect the second order nonlinear terms. The dc terms on both the sides are equal. The remaining first order linear ac terms are

$\hat{\imath}_{g}(t)=D \hat{\imath}(t)+I \hat{d}(t)$

Thus equations 15, 18, 20 are the small signal ac description of the ideal buck boost converter and are collected below:

$L\left(\frac{d \hat{\imath}}{d t}\right)=D \hat{v}_{g}(t)+D^{\prime} \hat{v}(t)+\left(V_{g}-V\right) \hat{d}(t)$

$c \frac{d \hat{v}(t)}{d t}=-D^{\prime} \hat{\imath}(t)-\frac{\hat{v}(t)}{R}+I \hat{d}(t)$

$\hat{\mathrm{i}}_{\mathrm{g}}(t)=D \hat{\imath}(t)+I \hat{d}(t)$

Equivalent circuit describing these three equations are now drawn one by one

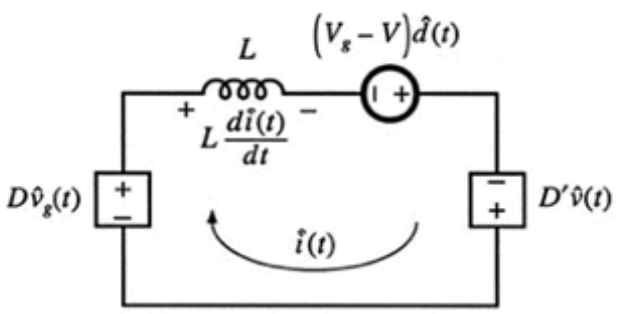

Fig 3 -Circvit for representing the small signal ac inductor loop equation

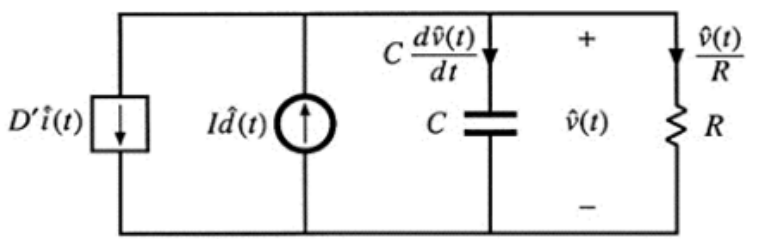

Fig 4 Circuit for representing the small signal ac capacitor node equation

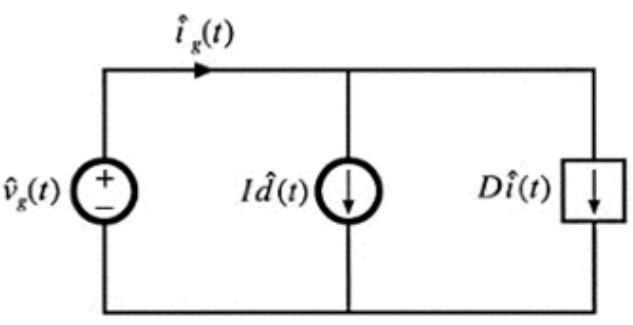

Fig 5 Circuit for representing the small signal ac input source equation transformer as shown in fig 6(b) the sinusoid superimposed imposed on transformer shows it is ideal and part of averaged small signal model.
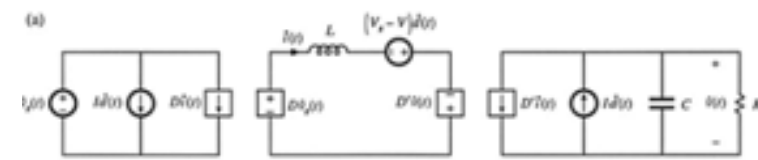

fig 6 a circuit for fig 3-5 collected together

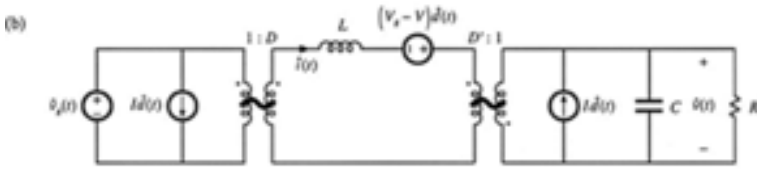

Fig $6 \mathrm{~b}$ small signal model obtained after combining dependent sources into transformer

This circuit is used to find converter transfer function, GM,PM and stability.

\section{III.STABILITY AND CONTROLLERS}

When a circuit is subjected to any disturbance because of change in duty cycle, input voltage or load and if the circuit comes to its steady state or regain its stability we call the circuit as stable one, but if circuit itself cannot regain stability we have to go for controllers. These controllers help to provide the circuit with stability.

The change in the output voltage with the variation in input load, current or duty cycle with frequency for switching converter is shown by developing it's dynamic model is called frequency response of converter circuit.

A bode plot is a plot of magnitude and phase of the transfer function as a function of frequency, where the magnitude is plotted in decibels and phase in degrees, respectively, while the frequency is shown on a logarithm scale. At a given frequency, the magnitude of product of two transfer functions is equal to the sum of the decibel magnitude of individual terms. Similarly, the phase of product of two transfer functions is equal to the sum of the phases of individual terms. This makes bode plot a simple and powerful tool to illustrate and calculate the loop gain parameters.

Assuming the gain magnitude of loop gain $\mathrm{T}(\mathrm{s})$ crosses unity $(0 \mathrm{~dB})$ only once, the system is stable if the phase lag at the crossover frequency is less than 180 degrees. At other frequencies, the phase lag may exceed 180 degrees and the system can be stable. The phase margin is the amount by which the phase lag at the crossover frequency is less than 180 degrees. The gain margin is the factor by which the gain is less than unity $(0 \mathrm{~dB})$ at the frequency where phase lag is 180 degrees. Typically, a phase margin of 45 degrees provides good response with little overshoot. In order to check for the stability of our Buck and Boost converter the circuit was implemented in Orcad Capture Pspice. VM and CM both are taken into consideration.

IV. IMPLIMENTATION OF SIMULATION MODELS In order to implement the small signal model of Buck and The circuits of fig 3--5 are collected in Fig6 (a), the Boost converter and to have its analysis done it is dependent sources can be combined into effective ideal necessary to have some circuit simulator. 
SPICE is an analog (originally) circuit simulator that was developed at the University of California at Berkeley. Pspice is developed by MicroSim Corporation which now taken over by Cadence ORCAD.

Most important advantage of PSpice's is that it helps the user simulate the circuit design graphically on the computer before building actual physical circuit. This helps the designer to make any necessary changes on the prototype without modifying any hardware or even before building it actually. Once the designer is complete with the test design, PSpice also help one run a check on it before deciding to come up with hardware model. Hence, PSpice allows one to check the functionality of the circuit model in real life simulations to confirm with his design. Since all the tests, designs and modifications are made over a terminal; the designer can save a lot of money that would have otherwise been spent on the building of models and modifying them.

\section{RESULT}

Simulation of VM Buck and Boost converter circuit with PWM switch without controller and its corresponding result:

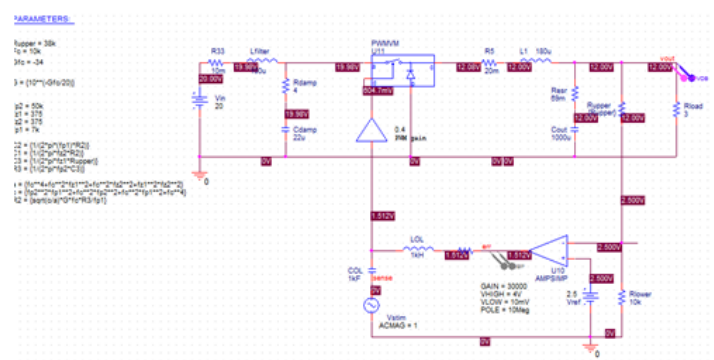

Fig 8 a Buck boost VM without controller

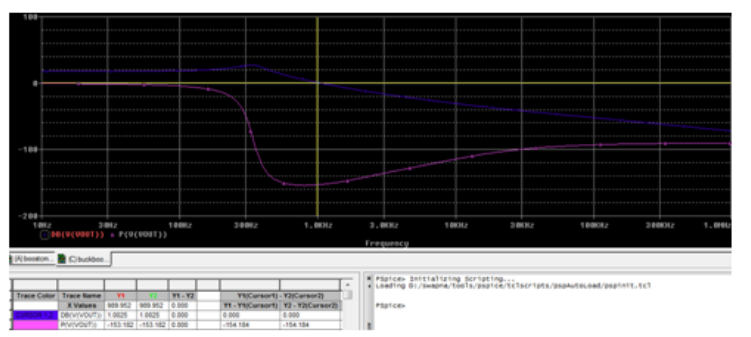

F ig $8 \mathrm{~b}$ Buck boost VM without controller power stage gain

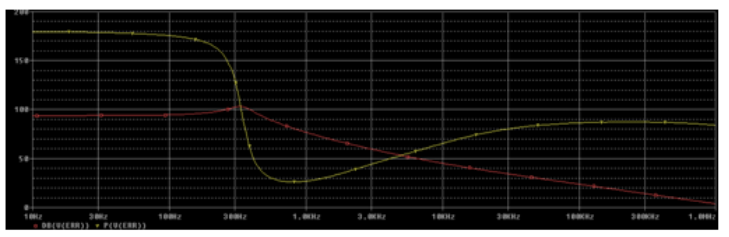

F ig $8 \mathrm{c}$ Buck boost VM without controller error signal

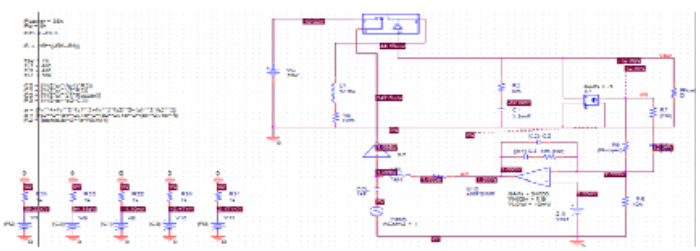

Fig 9 a Buck boost VM with type 3 compens ator
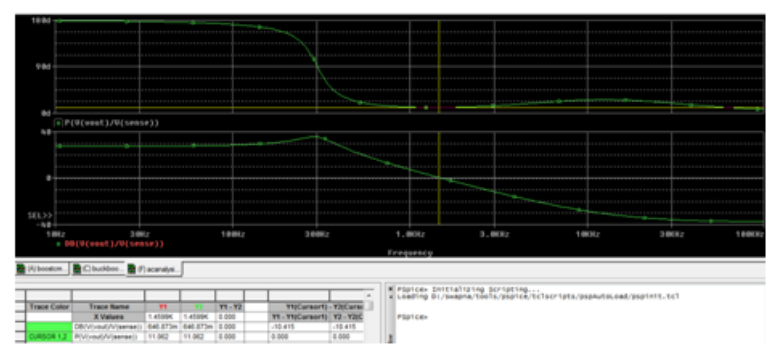

Fig $9 \mathrm{~b}$ Bode p lot for power stage alone for Buchboost VMconverter with type 3 comp ensator

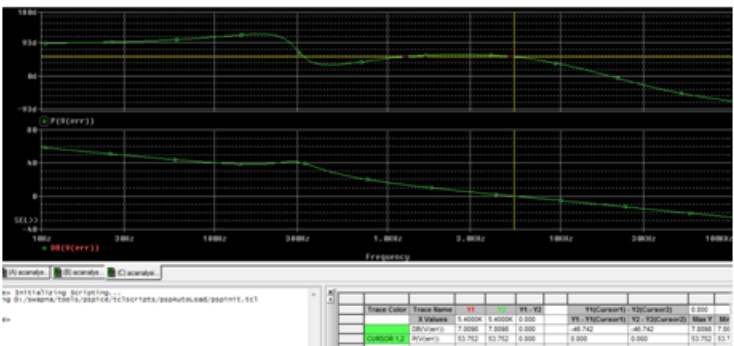

Fig $9 \mathrm{c}$ Bode plot for Buck boost type 3 VMafter applying final compensation

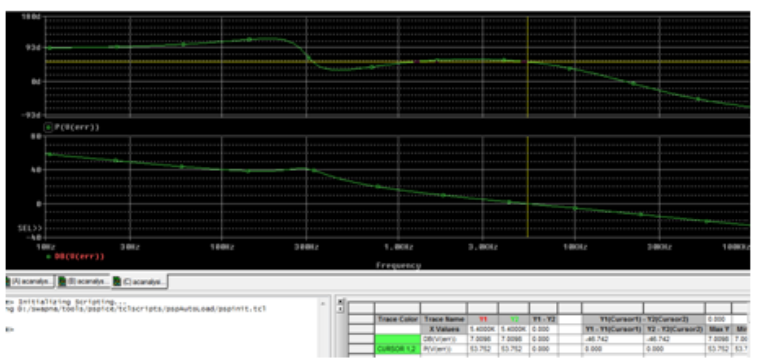

Fig $9 \mathrm{c}$ Bode plot for Buck boost type 3 VMafter applying final compensation

Similar analysis is also available for Buck and boost converter in CM control. A comparative table is prepared for VM case and for CM case independently. PM is calculated for each case i.e. Buck and boost converter in VM i) without controller and ii) With type 3 controller.

TABLE 1

Table 8.1: Table for comparison of various results obtained for small signal modeling of switch mode converters

\begin{tabular}{|c|c|c|c|c|c|}
\hline $\begin{array}{c}\text { S } \\
\text { No }\end{array}$ & $\begin{array}{c}\text { Fig } \\
\text { No }\end{array}$ & Type Of Converter & $\begin{array}{c}\text { Phase } \\
\text { Margin } \\
\text { Power } \\
\text { Converter }\end{array}$ & $\begin{array}{c}\text { Phase Margin } \\
\text { after } \\
\text { Compensation }\end{array}$ & $\begin{array}{c}\text { Gain Cross } \\
\text { Over } \\
\text { Frequency }\end{array}$ \\
\hline 1 & $8 \mathrm{8a}$ & $\begin{array}{c}\text { Buck boost VM PWM without } \\
\text { compensation }\end{array}$ & $-153.18^{\circ}$ & -- & $989.52 \mathrm{~Hz}$ \\
\hline 2 & $9 \mathrm{a}$ & $\begin{array}{c}\text { Buck boost VM PWM with type } \\
\text { 3 compensation }\end{array}$ & $-168.88^{\circ}$ & $53.75^{\circ}$ & $\mathbf{5 . 4} \mathrm{Khz}$ \\
\hline 3 & 1 & $\begin{array}{c}\text { Buck boost CM PWM with } \\
\text { type3 compensation }\end{array}$ & $\mathbf{9 5 . 9 3 ^ { \circ }}$ & $72^{\circ}$ & $\mathbf{5 1 6 . 7 5 \mathrm { Hz }}$ \\
\hline
\end{tabular}

\section{CONCLUSIONS}

From the results shown in fig $8 \mathrm{a}-9 \mathrm{c}$ and summary presented in table 8.1 it is clear that PM without controller for $\mathrm{VM}$ is very poor but with type 3 compensation not only positive but also greater than $45^{\circ}$ which is benchmark.

In case of $\mathrm{CM}$ controller with type 3 controller PM is positive and satisfactory. 


\section{REFERENCES}

[1] R. D. Middlebrook and S. Cuk, "A general unified approach to modeling switching-converter power stages," in Proc. ZEEE Power Electron. Specialists ConJ, 1976, pp. 18-34.

[2] V. Vorparian, "Simplified analysis of PWM converters using model of PWM switch, part I\&II: Continuous conduction mode," IEEE Trans. Aerosp Electron. System, vol. 26, no. 3, pp. 49M96, 1990.

[3] Amran. Y.. Huliehel, F., and Ben-Yaakgv, S. "A unified SPICE compatible averagemodel of PWM converters" IEEE Transactions on Power Electronics1991, 6, 585-594

[4] Keng C. Wu,"A Comprehensive Analysis of Current-Mode Control for DCM Buck-Boost Converters", IEEE transactions on industrial electronics, vol. 51, no. 3, June 2004.

[5] F. Guinjoan, A. Poveda, L. Martinez, L. G. Vicufi, J. Maj6, "An accurate small-signal modeling approach for switching dc-dc converters"1993 IEEE.

[6] Amir M. Rahimi, Parviz Parto, and Peyman Asadi "Compensator Design Procedure for Buck Converter with Voltage-Mode ErrorAmplifier'International Rectifier application note.

[7] APEC NCTU Taiwan, "Modeling and control of Dc-Dc converters" March 2013.Application note.

[8] Robert W. Erickson, Dragan Maksimovic , "Fundamentals of Power Electronics" Springer Science \& Business Media, 2001

[9] Christophe Basso, "Switch mode power supplies SPICE simulation and practical designs" Mc Graw Hill BPB publications first edition 2008. 